\title{
Simulation and Measurement Methods for RCS Estimations of a Scale Model Airplane
}

\author{
Naobumi Michishita ${ }^{1}$, Nguyen Quoc Dinh ${ }^{2}$, Yoshihide Yamada ${ }^{3}$ \\ ${ }^{1}$ Department of Electrical and Electronic Engineering, National Defense Academy, Japan \\ 2 Faculty of Radio-Electronics, Le Quy Don Technical University, Vietnam \\ 3 Department of Electronic Systems Engineering, Malaysia-Japan International Institute of Technology, \\ Universiti Teknologi Malaysia, Malaysia
}

Correspondence: Nguyen Quoc Dinh, dinhnq@mta.edu.vn

Manuscript communication: received 12 May 2015, accepted 28 September 2015

\begin{abstract}
In a military context, the radar cross section (RCS) of an airplane is a very important subject. For an RCS estimation object, a scaled-down model is often employed for ease of measurements. Recently, electromagnetic simulations of the RCS has become very convenient because of developments in high-speed calculation methods such as MLFMM and HOBF in electromagnetic simulators employing the MoM algorithm. As for the measurements, high-resolution measurements via the time domain analysis are promising. In this paper, important estimation expressions for use with a scale model are briefly explained. In terms of calculations, comparisons are drawn between the actual calculation abilities of MoM, MLFMM, and HOBF at $18 \mathrm{GHz}$ for a 1/48-scale model. In terms of measurement, a high-resolution measurement system employing a compact range and time domain analysis that is used in this study is explained. Next, the calculated and measured results are compared in order to show the accuracy of the obtained results. The results in vertical and horizontal planes of a scale model are shown at vertical and horizontal polarizations. Moreover, using the calculation method to determine estimates at higher frequencies is also investigated in order to obtain the practical RCS value of a real-sized airplane.
\end{abstract}

Keywords- radar cross section, scale model airplane, method of moment, compact range measurement, time domain analysis.

\section{INTRODUCTION}

The estimations and reductions of radar cross sections (RCS) are very important for designing military stealth airplanes. Much research has been reported on estimating monostatic RCS by employing scale models of perfect electrical conductor (PEC) structures [1, 2]. Highperformance algorithms with the method of moment (MoM), such as the multilevel fast multipole method (MLFMM) [3] and higher-order basis functions (HOBF), were utilized for calculating the RCS $[4,5]$. However, the calculation abilities were insufficient for estimating a real-sized airplane in the X-band using a personal computer. In this case, a ray-tracing method [equivalent to a geometrical optics (GO) method] was employed [6]. As for measurements, a compact range facility and high-resolution measurement utilizing a time domain analysis of a vector network analyzer were shown to be promising $[7,8]$.

In this paper, we first provide an overview of a scale model and show the influence of a scale factor on RCS values. Next, the calculation abilities of MoM, MLFMM, and HOBF in a FEKO simulator [9] are investigated by obtaining calculated data from a personal computer for a 1/48-scale model at $18 \mathrm{GHz}$. As for measurement, brief explanations of our measurement system, which employs a compact range and vector network analyzer, are provided. Then, the measured and calculated data are compared to show the accuracies of achieved results. Moreover, in order to increase the estimation frequency from $18 \mathrm{GHz}$ to $144 \mathrm{GHz}$, a geometrical optics (GO) calculation method is employed.

\section{RCS Expressions for a ScAle Model}

The configuration of a scale model for calculation is shown in Figure 1. Here, airplanes are composed of PEC surfaces. When the size of an object is reduced to $1 / \alpha$ according to miniaturizations of mesh sizes to $1 / \alpha$, the estimation frequency should be increased $\alpha$ times. Then, the angular responses of the RCS results become identical between a real object and a scale model. However, the RCS value $(\sigma)$ itself is reduced by the scale factor of $1 / \alpha^{2}$. In order to realize the above mentioned RCS $(\sigma)$ characteristics, $\sigma$ is explained through the following equations.

In the case of Figure 1(a), $\sigma$ is defined as

$$
\sigma=4 \pi R^{2} \frac{\left|E_{\mathrm{r}}\right|^{2}}{\left|E_{\mathrm{i}}\right|^{2}}
$$




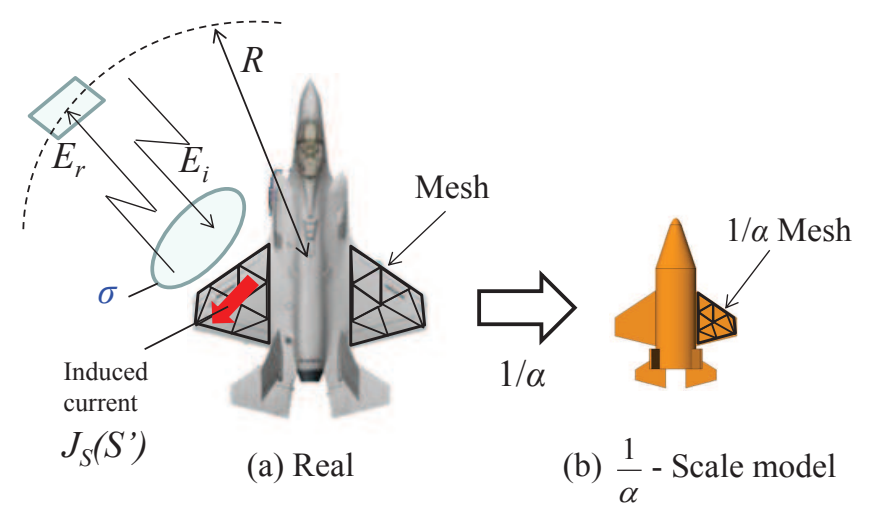

Figure 1. Scale model configuration.

The reflected electric field $\left(E_{\mathrm{r}}\right)$ is given by

$$
\begin{aligned}
E_{\mathrm{r}} & =\frac{-j \omega \mu e^{-j k R}}{4 \pi R} \iint_{S} J_{S}\left(S^{\prime}\right) e^{j k r^{\prime}} d s^{\prime} \\
& =\frac{-j k \eta}{4 \pi R} e^{-j k R} \iint_{S} J_{S}\left(S^{\prime}\right) e^{j k r^{\prime}} d s^{\prime} .
\end{aligned}
$$

Then, $\sigma$ can be expressed by

$$
\sigma=\frac{k^{2} \eta^{2}}{4 \pi} \frac{\left|\iint_{S} J_{S}\left(S^{\prime}\right) e^{j k r^{\prime}} d s^{\prime}\right|^{2}}{\left|E_{\mathrm{i}}\right|^{2}} .
$$

Here, $J_{\mathrm{s}}$ is related to $E_{\mathrm{i}}$ according to

$$
J_{S}=2 H_{\mathrm{i}}=2 E_{\mathrm{i}} / \eta
$$

Then, the integral part becomes $\left(E_{\mathrm{i}} S\right)^{2}$ in the case of a flat plate having area $S$. Therefore, for the flat plate, $\sigma$ is given by the following expression:

$$
\sigma=\frac{4 \pi S^{2}}{\lambda^{2}}
$$

In the case of a scale model having a scale factor of $1 / \alpha, \lambda^{\prime}$ becomes $\lambda / \alpha$ and $S^{\prime}$ becomes $S / \alpha^{2}$. When these values are inserted in Equation (5), the RCS value $\left(\sigma^{\prime}\right)$ of a scale model is obtained by

$$
\sigma^{\prime}=\frac{4 \pi S^{\prime 2}}{\lambda^{\prime 2}}=\frac{4 \pi S^{2}}{\lambda^{2}} \frac{1}{\alpha^{2}} .
$$

It should be noted that the RCS value is reduced by a scale factor of $1 / \alpha^{2}$ in the case of a scale model.

\section{Calculation Methods}

\subsection{Structure of a Calculation Model}

The calculation structure is shown in Figure 2. This structure is a simplified model of a jet fighter. The canopy and the intake of the jet engine are eliminated. The wing and tail sections are composed of flat plates for ease of fabrication. All surfaces are perfect conductors. The scale-down ratio is $1 / 48$. The length of the model becomes $310 \mathrm{~mm}$. This size is very convenient to handle for measuring purposes.

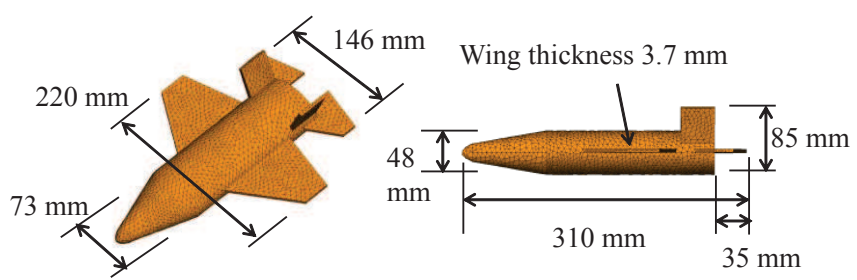

(a) Bird's eye view

(b) Side view

Figure 2. Structure of $1 / 48$ scale model.

Table I

Employed Simulation Methods

\begin{tabular}{|l|c|l|}
\hline \multicolumn{1}{|c|}{ Method } & Mesh size & \multicolumn{1}{c|}{ Concept } \\
\hline $\begin{array}{l}\text { Method of } \\
\text { Moment (MoM) }\end{array}$ & $\begin{array}{l}\text { Less } \\
\lambda / 10\end{array}$ & $\begin{array}{l}\text { Exact induced currents are calculated } \\
\text { Matrix equation should be solved }\end{array}$ \\
\hline $\begin{array}{l}\text { Multilevel fast } \\
\text { multipole method } \\
\text { (MLFMM) }\end{array}$ & $\begin{array}{l}\text { Less } \\
\lambda / 10\end{array}$ & $\begin{array}{l}\text { Simplification of matrix } \\
\text { equation by grouping of } \\
\text { far aparted points }\end{array}$ \\
\hline $\begin{array}{l}\text { Higher order } \\
\text { basis functions } \\
\text { (HOBF) }\end{array}$ & $\approx \lambda$ & $\begin{array}{l}\text { Expression of current } \\
\text { distributions by higher } \\
\text { order basis functions }\end{array}$ \\
\hline $\begin{array}{l}\text { Geometrical optic } \\
\text { method (GO) }\end{array}$ & $\approx \lambda$ & $\begin{array}{l}\text { Ray tracing } \\
\text { Reflection law }\end{array}$ \\
\hline
\end{tabular}

\subsection{Simulation Methods}

The simulation methods employed in this paper are summarized in Table I. MoM is a basic method for precisely calculating induced currents on object surfaces. However, a very large computation memory is required because we are using a very small mesh size of less than $1 / 10$ wavelengths $(\lambda)$ for the objective surfaces. MLFMM is an approximation method of MoM that simplifies calculation processes between points that are far apart. Therefore, computation memory size is reduced. HOBF is also an approximation method of MoM that employs a higher-order basis function in MoM calculations. In this case, a large mesh size such as 1 wavelength $(\lambda)$ can be permitted. Hence, we can expect extreme savings in computation memory and computational time. GO is the most simple calculation method; it employs a ray-tracing method for the objective surfaces. Diffractions on the objective surfaces are estimated based on the law of reflection. Thus, GO can be reliable only when applied to simple surface configurations. However, for very large objects, only this method is applicable.

\subsection{Practical Performances of Simulation Methods}

Monostatic RCS calculations are performed by MoM, MLFMM, and HOBF algorithms using a FEKO simulator [9]. Table II shows the required computer resources. Here, a frequency of $18 \mathrm{GHz}$ is selected by taking into account the measurement conditions. In the basic MoM, a small mesh size of 0.1 wavelengths is needed. In addition, a very large-scale matrix equation having 124,740 unknowns should be solved. The calculation memory becomes $116.336 \mathrm{~GB}$, and the required calculation time is 78 hours. In the case of MLFMM, calculation 
Table II

Required Computer Resources

\begin{tabular}{|c|c|c|c|c|c|}
\hline $\begin{array}{c}\text { Algo- } \\
\text { rithm }\end{array}$ & $\begin{array}{c}\text { Mesh } \\
\text { size }\end{array}$ & $\begin{array}{c}\text { Mesh } \\
\text { number }\end{array}$ & $\begin{array}{c}\text { Unknown } \\
\text { number }\end{array}$ & $\begin{array}{c}\text { Memory } \\
\text { amount }\end{array}$ & $\begin{array}{c}\text { Calculation } \\
\text { time }\end{array}$ \\
\hline MoM & $0.1 \lambda$ & 83,160 & 124,740 & $\begin{array}{c}116.336 \\
\text { GB }\end{array}$ & $\begin{array}{c}282,293 \\
\text { seconds } \\
(78 \text { hours })\end{array}$ \\
\hline MLFMM & $0.1 \lambda$ & 83,160 & 124,740 & $\begin{array}{c}5.430 \\
\text { GB }\end{array}$ & $\begin{array}{c}19,999 \\
\text { seconds } \\
(5.6 \text { hours })\end{array}$ \\
\hline HOBF & $\lambda$ & 790 & 14,207 & $\begin{array}{c}1.527 \\
\text { GB }\end{array}$ & $\begin{array}{c}4,168 \\
\text { seconds } \\
(1.2 \text { hours })\end{array}$ \\
\hline
\end{tabular}

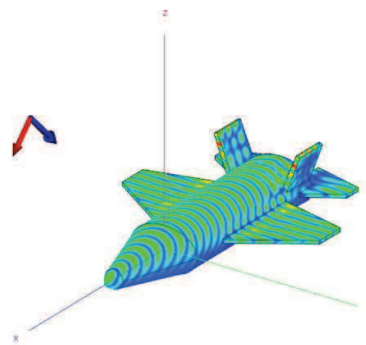

(a) $\operatorname{MoM}(\operatorname{Mesh}=\lambda / 10)$

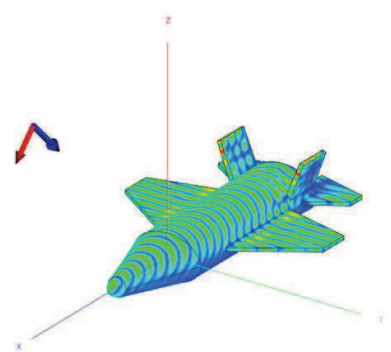

(b) $\operatorname{MLFMM}(\operatorname{Mesh}=\lambda / 10)$

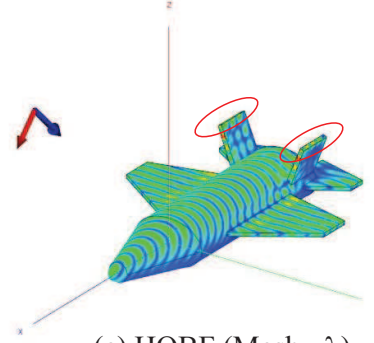

(c) $\operatorname{HOBF}(\mathrm{Mesh}=\lambda)$

Figure 3. Current distribution.

memory size is reduced to 5.4 GB. Calculation time is also reduced, to $5.6 \mathrm{~h}$. In the case of HOBF, a large mesh size of $\lambda$ can be allowed. Hence, calculation memory is reduced to $1 / 3.5$, and the calculation time is reduced to $1 / 4.5$ compared to those of MLFMM. Thus, HOBF is the most suitable algorithm for RCS calculations because the calculation results of the three methods are almost the same as shown followings.

RCS is calculated from induced currents on the object as shown in Equation (3). So, comparing current distributions on a scale model may be most adequate in order to show calculation accuracies. The induced current distributions of MoM, MLFMM and HOBF are shown in Figure 3. In the case of HOBF calculation, 3.5-th higher order function is used. Three current distributions agree very well. Only slight differences are observed in $\mathrm{HOBF}$ at the edges of rear vertical wings indicated by small circles. As a result, calculation accuracies of three methods are shown very well.

\section{Measurement Method}

\subsection{Measurement Setup}

The RCS measurement configuration is shown in Figure 4 . As one feature of this setup, a compact range
Table III

Features of MeAsurement Setur

\begin{tabular}{|l|l|l|}
\hline \multicolumn{1}{|c|}{ Item } & \multicolumn{1}{c|}{ Contents } & \multicolumn{1}{c|}{ Notes } \\
\hline Frequency & $12 \mathrm{GHz}$ to $19 \mathrm{GHz}$ & Wide sweep range \\
\hline Horns & Transmit/Receive & Coupling to receive horn \\
\hline Data analysis & Time domain & Target pulse is selected \\
\hline Set up & Compact range & Place target in near point \\
\hline
\end{tabular}

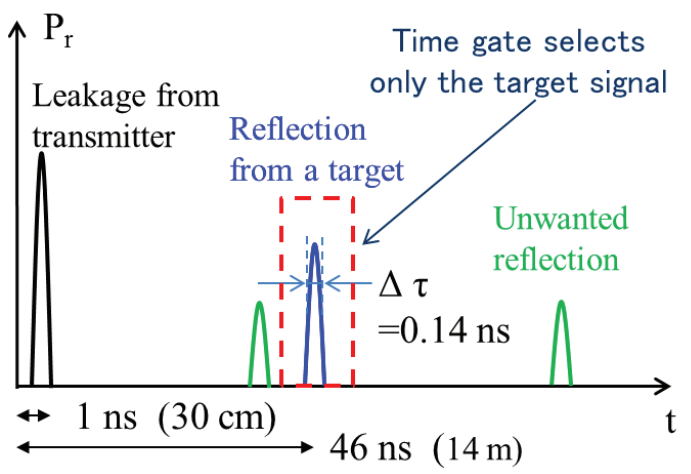

Figure 5. Display indications of time domain pulses.

configuration from an offset parabolic reflector is used. Thus, far-field RCS characteristics can be obtained by using a very close arrangement of a target and measurement horns. Another feature of the measurement configuration is the employment of a vector network analyzer (VNA). During transmission, the frequency is swept from $12 \mathrm{GHz}$ to $19 \mathrm{GHz}$. During reception, at each rotated angle $\theta_{i}(i=1,2, \ldots)$, the RCS levels of the swept frequency are obtained. Then the swept frequency intensities are converted to time domain signals by the Fourier transformation function. The time domain pulse signals are indicated on a PC display. Features of this setup are summarized in Table III. Usually, the adjacent placement of transmit and receive horns is not recommended because of large coupling from the transmit horn to the receive horn. However, in this setup, this coupling can be omitted by selecting only the reflected pulse in the time domain signals. At the same time, all interference from surrounding obstacles is also omitted. As a result, the time domain selection process greatly increases the measurement dynamic range. Results of the high-resolution measurement will be shown in the following sections.

\subsection{Time Domain Characteristics}

The resultant time domain signals from a PC display are shown in Figure 5. All received waves are arranged in shapes of pulses on the time axis depending on their differences in arrival time. Each pulse has a $3 \mathrm{~dB}$ time spread of $\Delta \tau$. When the frequency sweep range is expressed by $\Delta f$, the relation between $\Delta \tau$ and $\Delta f$ is given by the next expression:

$$
\Delta \tau=\frac{1}{\Delta f} .
$$




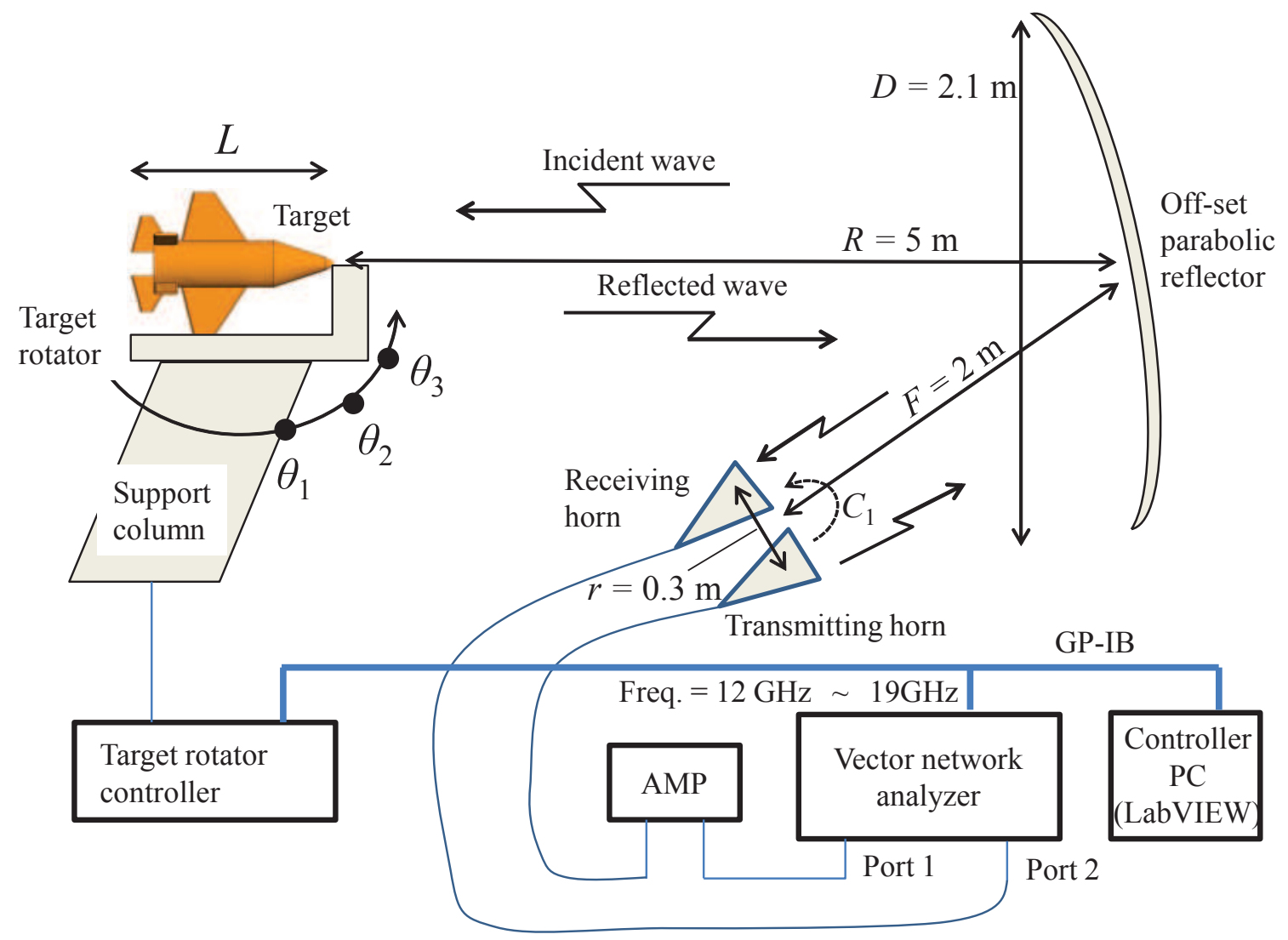

Figure 4. Experimental setup.

In the measurement, $\Delta f$ becomes $7 \mathrm{GHz}$. Then, $\Delta \tau$ becomes $0.14 \mathrm{~ns}$. In addition, a time period of $0.14 \mathrm{~ns}$ corresponds to the wave propagation distance of $4.2 \mathrm{~cm}$. As shown in Figure 5, the pulse reflected by the target is placed at $46 \mathrm{~ns}$ in the time axis. The pulse coupled by the transmitting horn is placed at $1 \mathrm{~ns}$. Therefore, by selecting the target signal with the time gate function, the influence of all surrounding reflected waves is deleted. As a result, a high-resolution measurement is achieved.

\subsection{Calculated and Measured Results}

Comparisons of calculated and measured RCS results at $18 \mathrm{GHz}$ for the vertical and horizontal planes are shown in Figures 6 and 7, respectively. Here, polarization characteristics such as the vertical and horizontal polarizations are examined. In calculation, the HOBF algorithm is used. In Figure 6(a) of the vertical polarization, measured and calculated results agree very well, from a large value of $9 \mathrm{dBsm}$ to a small value of $-40 \mathrm{dBsm}$, in all directions. Here, dBsm indicateds a $\mathrm{dB}$ square meter. Only in the top direction $0^{\circ}$ does the measured result become too small to the simulated value of $9.6 \mathrm{dBsm}$. The reason for this decrease in the measurement value is the insufficient alignment of the measured model setting. In the case of the horizontal polarization of Figure 6(b), effects of misalignment in the vertical plane of the scale model setup become remarkable. At near $0^{\circ}$ and $180^{\circ}$, the measured values become lower than the calculated values. However, in other directions, the measured values agree rather well with the calculated values. It should be noted that alignment in the vertical plane is very sensitive at the horizontal polarization measurement. In the case of the horizontal plane of Figure 7(a) and 7(b), the measured and calculated results agree very well, from $-4 \mathrm{dBsm}$ to $-40 \mathrm{dBsm}$, in all directions. As a result, high-resolution and reliable results are achieved in this measurement.

\section{Increase of Calculation Frequency}

\subsection{Calculation Abilities at $70 \mathrm{GHz}$}

RCS values at higher frequencies are required in order to estimate actual RCS values. Thus, the exacting method of HOBF and the simplest method of GO are investigated at $70 \mathrm{GHz}$. In Table IV, the calculation resources are shown.

For HOBF, a very large computer memory of 199.8 GB is required. This is nearly the limit of our PC. Moreover, the calculation time becomes $190 \mathrm{~h}$. In the case of GO, the required computer memory is very small. However, the calculation time becomes $18 \mathrm{~h}$. In Figures 8 and 9, the angular responses of RCS on the vertical and the horizontal planes are compared, respectively. In the vertical plane of Figure 8 , the GO results agree very well with the $\mathrm{HOBF}$ results except in the front direction. In the angular region from 100。 to $150^{\circ}$, the GO results become smaller than the HOBF results. In this direction, the RCS of a tapered shape 


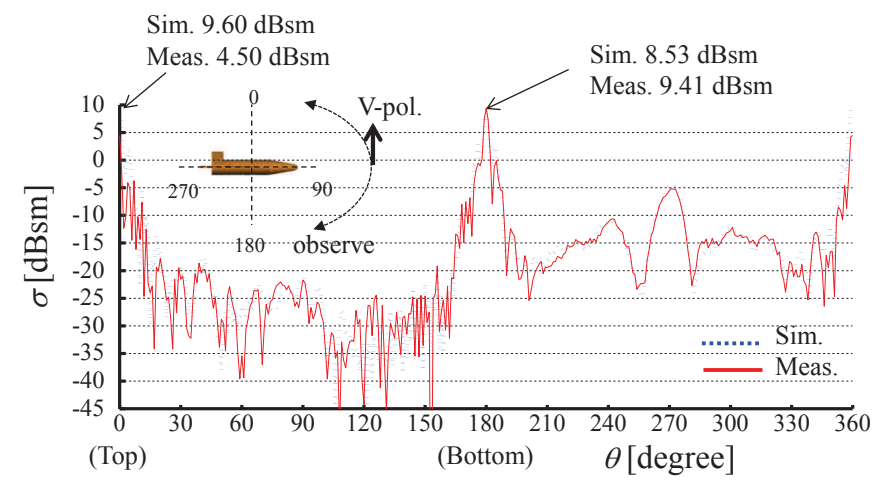

(a) Vertical polarization

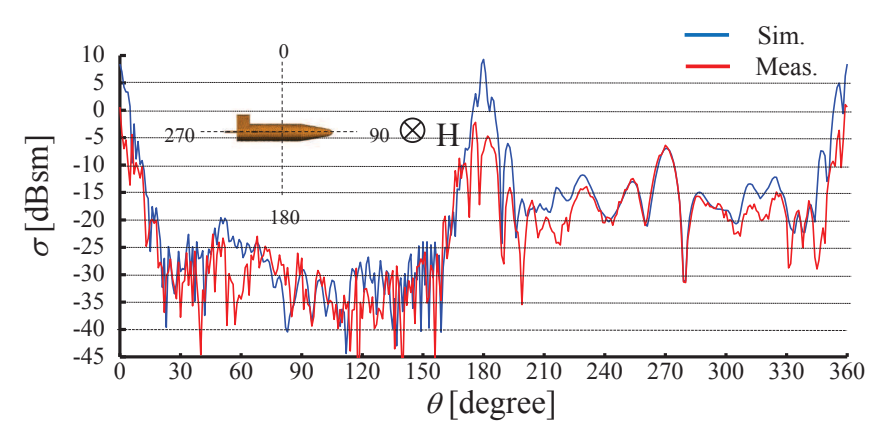

(b) Horizontal polarization

Figure 6. Monostatic RCS of Simulation versus Measurement in the vertical plane.

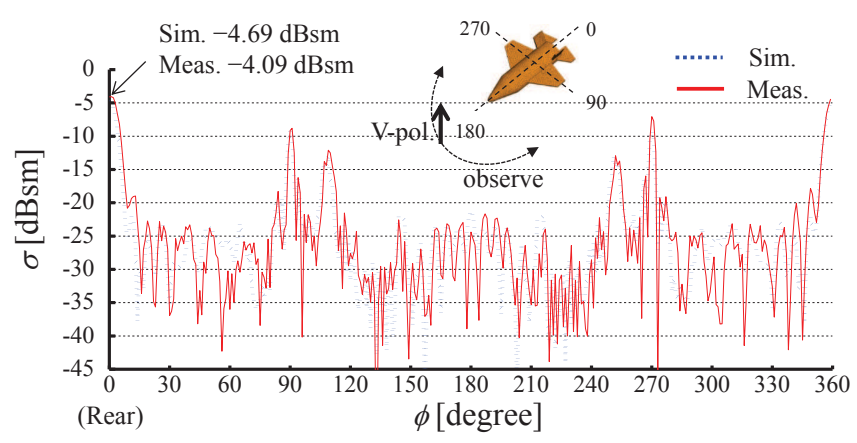

(a) Vertical polarization

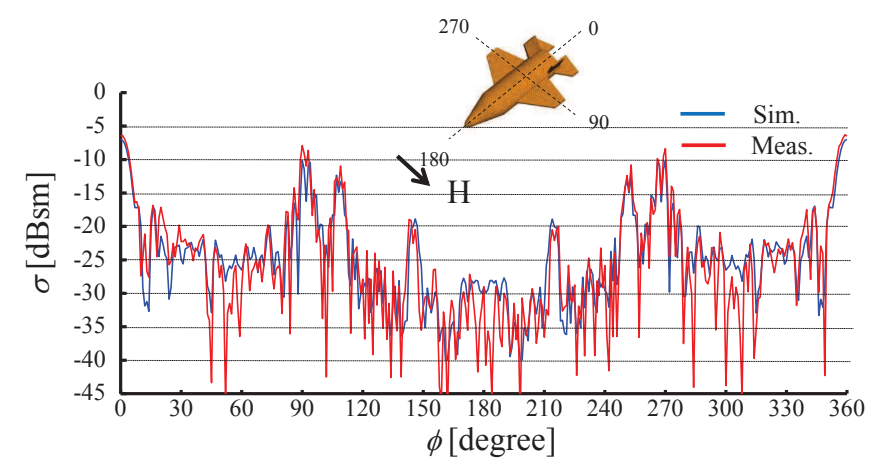

(b) Horizontal polarization

Figure 7. Monostatic RCS of Simulation versus Measurement in the horizontal plane.

becomes dominant. In Figure 9, GO results become smaller than $\mathrm{HOBF}$ results from $30^{\circ}$ to $80^{\circ}$ and from $280^{\circ}$ to $330^{\circ}$. These angular regions correspond to reflections from the vertical and tail wings. We noted that
Table IV

Computational Data at $70 \mathrm{GHz}$

\begin{tabular}{|c|c|c|c|c|c|}
\hline $\begin{array}{c}\text { Algo- } \\
\text { rithm }\end{array}$ & $\begin{array}{c}\text { Mesh } \\
\text { size }\end{array}$ & $\begin{array}{c}\text { Mesh } \\
\text { number }\end{array}$ & $\begin{array}{c}\text { Unknown } \\
\text { number }\end{array}$ & $\begin{array}{c}\text { Memory } \\
\text { amount }\end{array}$ & $\begin{array}{c}\text { Calculation } \\
\text { time }\end{array}$ \\
\hline MLFMM & \multicolumn{6}{|c|}{ Calculation is impossible } \\
\hline HOBF & $\lambda$ & 9,092 & 163,656 & $\begin{array}{c}199,811 \\
\text { GB }\end{array}$ & $\begin{array}{c}684,574 \\
\text { seconds } \\
(190 \text { hours })\end{array}$ \\
\hline GO & $\lambda$ & 12,536 & & $\begin{array}{c}91,466 \\
\text { MB }\end{array}$ & $\begin{array}{c}64,716 \\
\text { seconds } \\
(18 \text { hours })\end{array}$ \\
\hline
\end{tabular}

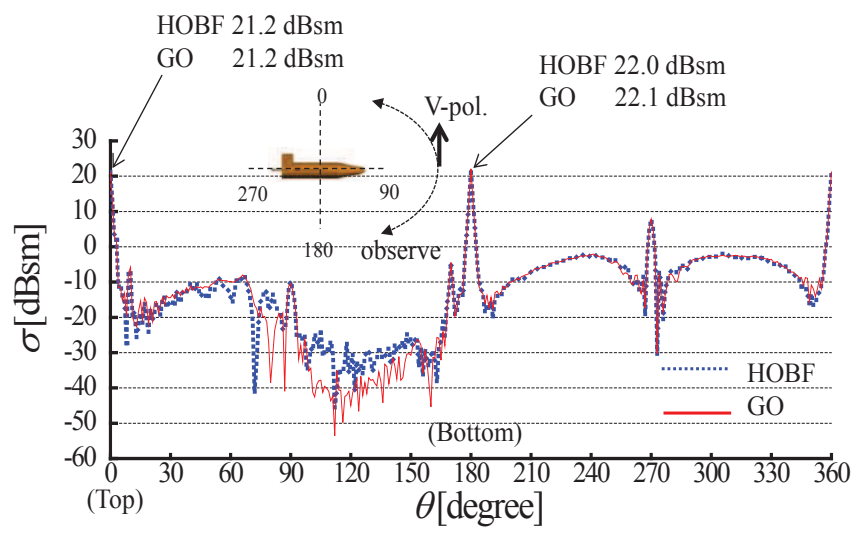

Figure 8. Monostatic RCS in the vertical plane.

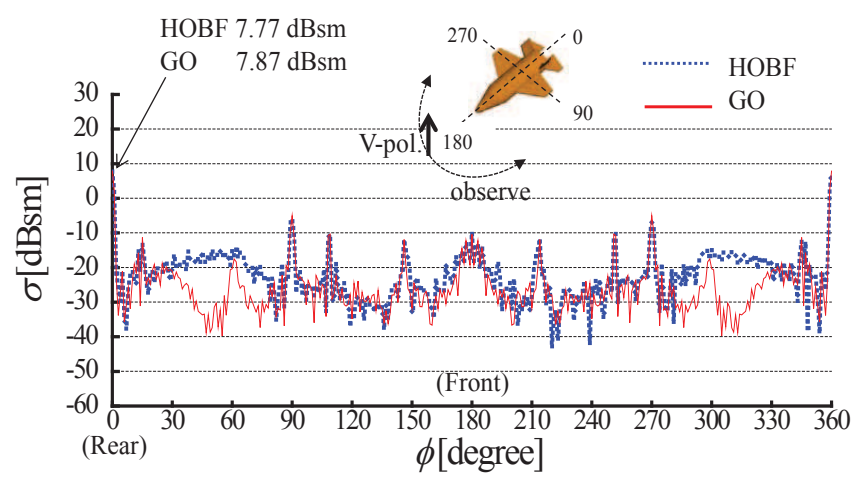

Figure 9. Monostatic RCS in the horizontal plane.

rather complicated diffractions arose in these angular regions.

As a result, GO can be used for rough estimations except in directions that result in special reflections and diffractions.

\subsection{GO Calculations in Increased Frequencies}

In order to understand the computational difficulties in attempting to increase the calculation frequency, computer memory sizes and calculation times are shown in Figure 10. HOBF reaches its limit of memory size at $70 \mathrm{GHz}$. At higher frequencies, GO can be applicable. Although the required memory is very small, large calculation times are required. At $96 \mathrm{GHz}$, which corresponds to $2 \mathrm{GHz}$ in a real airplane, the calculation time becomes $64 \mathrm{~h}$. At $144 \mathrm{GHz}$, which corresponds to $3 \mathrm{GHz}$ in a real airplane, the calculation time becomes $314 \mathrm{~h}$. This frequency appears to be the 


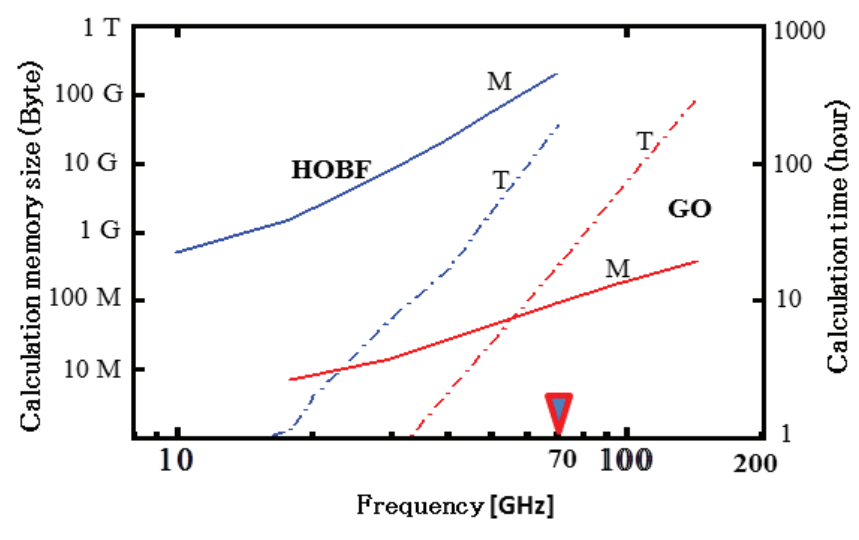

Figure 10. Computational limits.

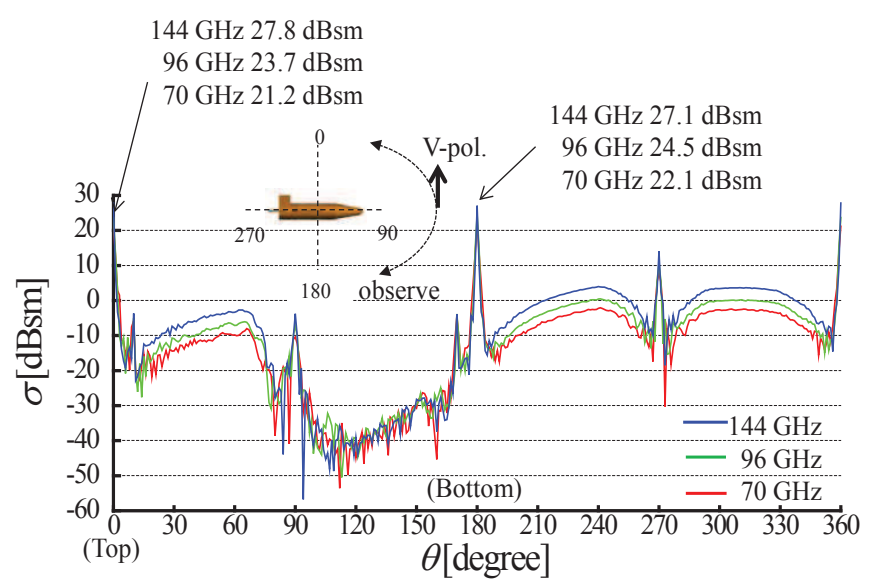

Figure 11. Monostatic RCS in the vertical plane.

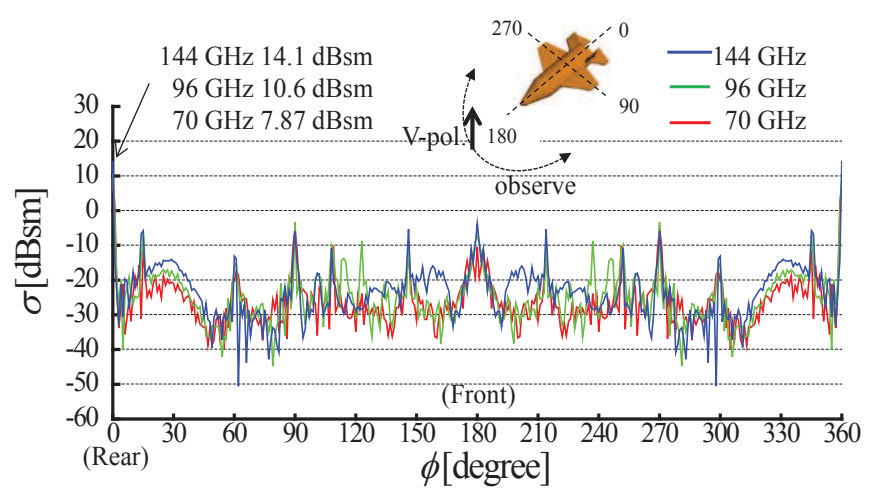

Figure 12. Monostatic RCS in the horizontal plane.

highest achievable value in the present electromagnetic simulation as operated on a personal computer.

Calculated GO results are shown in Figures 11 and 12 in the vertical and the horizontal planes, respectively. In the vertical plane of Figure 11, at angular regions of $20^{\circ} \sim 70^{\circ}, 190^{\circ} \sim 260^{\circ}$ and $280^{\circ} \sim 350^{\circ}, \sigma$ values change very slowly for angular change and increase in accordance with the frequency increase. However, in the nose direction of $70^{\circ} \sim 160^{\circ}, \sigma$ values become very small and have no frequency dependence. Because the structure corresponding to this direction is a tapered cone, frequency dependences does not occur.

In the horizontal plane of Figure 12, at the rear directions from $0^{\circ}$ to $50^{\circ}$ and from $310^{\circ}$ to $360^{\circ}$, frequency dependences of $\sigma$ become adequate. In the other angular regions, $\sigma$ changes during frequency increases, do not perform according to standard rules. In these angular regions, complicated reflections and diffractions are related and unusual frequency characteristics occur.

As a result of GO calculations, it is shown that RCS calculations by PC is possible up to the frequency $144 \mathrm{GHz}$. However, calculated RCS values should be treated as a reference.

\section{Conclusion}

Simulations and measurement are achieved at $18 \mathrm{GHz}$ for a model airplane with a downsized scale of $1 / 48$. In the simulation, a personal computer is employed. In this case, the fast calculation algorithm HOBF of the MoM method is shown to be effective. During measuring, the introduction of the time domain function of a vector network analyzer is shown to achieve high dynamic range measurement. A very good agreement between the calculated and measured results is obtained. Hence, the accuracy of the calculated and measurement methods is ensured. Moreover, in order to obtain simulated results at higher frequencies, GO calculations are studied. It is shown by a personal computer that the simulation limit is $144 \mathrm{GHz}$.

\section{ACKNOWLEDGMENT}

The authors would like to thank Dr. Toshiharu Itoh and Mr. Kazuya Matsubayashi of the Japan Ministry of Defense for their help with using the RCS measurement system.

\section{REFERENCES}

[1] B. Jacobs and D. Baker, "Validation of a computational electromagnetic model of a boeing 707 aircraft by comparison to scale model measurements," in IEEE-APS Topical Conference on Antennas and Propagation in Wireless Communications (APWC). Cape Town, South Africa: IEEE, Sept. 2012, pp. 1001-1004.

[2] A. B. Gorji, R. C. Janalizadeh, and B. Zakeri, "RCS computation of a relatively small complex structure by asymptotic analysis," in Proceedings of 2013 URSI International Symposium on Electromagnetic Theory (EMTS). Hiroshima, Japan: IEEE, May 2013, pp. 1066-1069.

[3] J. Song, C.-C. Lu, and W. C. Chew, "Multilevel fast multipole algorithm for electromagnetic scattering by large complex objects," IEEE Transactions on Antennas and Propagation, vol. 45, no. 10, pp. 1488-1493, Oct. 1997.

[4] B. Kolundzija, M. Tasic, D. Olcan, D. Zoric, and S. Stevanetic, "Full-wave analysis of electrically large structures on desktop pcs," in Computational Electromagnetics International Workshop (CEM). Izmir, Turkey: IEEE, Aug. 2011, pp. 122-127.

[5] Y. Yan, Y. Zhang, C.-H. Liang, H. Zhao, and D. GarcíaDoñoro, "RCS computation by parallel mom using higher-order basis functions," International Journal of Antennas and Propagation, vol. 2012, pp. 1-8, 2012.

[6] F. Weinmann, "Ray tracing with po/ptd for rcs modeling of large complex objects," IEEE Transactions on Antennas and Propagation, vol. 54, no. 6, pp. 1797-1806, 2006. 
[7] M. W. Shields and A. J. Fenn, "A new compact range facility for antenna and radar target measurements," Lincoln Laboratory Journal, vol. 16, no. 2, pp. 381-391, 2007.

[8] Y. Yamada, N. Michishita, and Q. D. Nguyen, "Calculation and measurement methods for rcs of a scale model airplane," in IEEE International Conference on Advanced Technologies for Communications (ATC), 2014, pp. 69-72.

[9] FEKO suite 6.3 comprehensive electromagnetic solutions. www.feko.info.

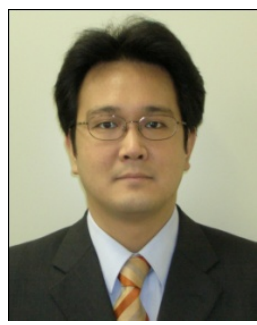

Naobumi Michishita received the B.E., M.E. and D.E. degrees in electrical and computer engineering from Yokohama National University, Yokohama, Japan, in 1999, 2001, and 2004, respectively. In 2004, he was a research associate at the Department of Electrical and Electronic Engineering, National Defense Academy, Kanagawa, Japan, where he is currently an associate professor. From 2006 to 2007 , he was a visiting scholar at the University of California, Los Angeles. His current research interests include metamaterial antenna and electromagnetic analysis.

$\mathrm{He}$ is a member of the Institute of Electronics, Information and Communication Engineers (IEICE), Japan. He is also members of the Japan Society for Simulation Technology and the Institute of Electrical and Electronics Engineers (IEEE). He was the recipient of the Young Engineer Award presented by the IEEE Antennas and Propagation Society Japan Chapter and the IEICE, Japan (2004 and 2005). He received the best paper award and the best tutorial paper award from the IEICE Communication Society in 2013 and 2014, respectively.

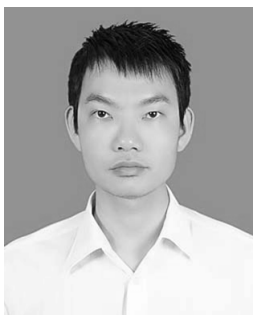

Nguyen Quoc Dinh received the B.E., M.E and D.E. degrees in Department of Electrical \& Electronic Engineering, National Defense Academy, Yokosuka, Japan, in 2006, 2008, and 2011, respectively. Since 2011, he has been a Research Associate at the Faculty of RadioElectronics, Le Quy Don Technical University, Hanoi, Vietnam. His research interests include very small antennas, UWB antennas, MIMO antennas, RCS. He is a member of the Institute of Electronics, Information and Communication Engineers (IEICE), Japan. He was the recipient of the Young Scientist Award by Technical Group on Antennas and Propagation of the IEICE, Japan (2011).

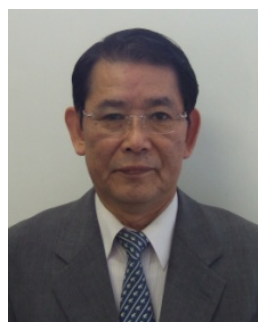

Yoshihide Yamada received the B.E. and M.E. degrees on electronics from Nagoya Institute of Technology, Nagoya, Japan in 1971 and 1973, respectively. And he received the D.E. degree on electrical engineering from Tokyo Institute of Technology, Tokyo, Japan in 1989. In 1973, he joined the Electrical Communication Laboratories of Nippon Telegraph and Telephone Corporation (NTT). Till 1984, he was engaged in research and development of reflector antennas for terrestrial and satellite communications. From 1985, he engaged in R\&D of base station antennas for mobile radio systems. In 1993, he moved to NTT Mobile Communications Network Inc. (NTT DoCoMo). In 1995, he was temporarily transferred to YRP Mobile Telecommunications Key Technology Research Laboratories Co., Ltd. At the same time, he was a guest professor of the cooperative research center of Niigata University, and a lecturer of Science University of Tokyo, both from 1996 to 1997. In 1998, he changed his occupation to a professor of National Defense Academy, Kanagawa, Japan. In 2014, he has started working as a professor at Malaysia-Japan International Institute of Technology, Universiti Teknologi Malaysia, Kuala Lumpur, Malaysia. He received the best paper award and the best tutorial paper award from IEICE in 2013 and 2014, respectively. Now, he is interested in very small antennas, array antennas, aperture antennas and electromagnetic simulation of RCS. He is a Fellow member of the IEICE and is a senior member of IEEE AP society. He is also a member of the ACES. 